\title{
The Design Of Management System Of Micro Theater Chain
}

\author{
Jingming $\mathrm{Liu}^{1, \mathrm{a}}$, Sanxing $\mathrm{CaO}^{2, \mathrm{~b}}$ \\ ${ }^{1,2}$ School of Communication University of China Beijing 100024, China; \\ a648124083@qq.com, bcaosx_cuc@126.com
}

Keywords: Micro Film , Micro Theater Chain Devise , Offline Show , Database

\begin{abstract}
Micro film, as a new pattern of artistic dissemination, breaking away from restrictions and fetters during creating, can reflect the epitome of every director's dream within a short period of time. It's becoming more and more necessary to get a micro theater chain to provide offline opportunities to show, to market and to disseminate numerous micro films. In this project, we mainly study a service, which provides reference and selection for micro films offline projecting, depending on the China Video Exchange and Collabrations. The aim of this project is to lead audiences to exploit the micro film projecting information the micro theater chain provides to watch micro films, to make it convenient, to create various modes for watch films, emphasizing the audience is the master, and to realize the purpose of audiences' entertaining consumption. For techniques, HTML, CSS, PHP, JAVASCRIPT, MYSQL database are used to establish front platform and backstage management system.
\end{abstract}

\section{Introduction Of ChinaVEC}

ChinaVEC is China Video Exchange and Collabrations shorthand, Chinese name is "China micro video collaboration and trading platform." Trading platform ChinaVEC micro video collaboration platform that is China is a financial and trading platform work creation, investment, broadcast channels, talent development, coordination and innovation as one of the online and offline. The formation of micro films and micro-video field of high-quality resources and creativity of convergence, open up the bottleneck in the development of micro-film, coordination of all forces promoting the sound development of China's micro-micro-film and video. The platform can be achieved on micro-video upload, playback, micro film school, micro cinema, micro-video trade, micro-video activities, integrated communication system and so on[1]. ChinaVEC Platform as a Chinese micro-video collaboration and trading platform Home current total includes the following modules: Home, micro film school, topic micro-video program, micro-video purchases, offline, online communication, project management, and micro-micro-video video authorize a total nine. This time, the design is need to add a micro-cinema in the above modules.

\section{Micro-cinema system module front-end design and implementation}

\section{Database set up a data table}

In this management system, uses a MySQL database. Because of this design is dependent in ChinaVEC Platform, so only need to create data tables in the original database chinavec. For simplicity convenience and efficiency considerations, this system is designed to extend the establishment of only one data table, the data include the entire data table records all videos (id), director (director), producer (producer), Chinese title (title_cn), English title (title_en), video content Overview (dscrp), the film type (up_type), specific time screening (up_time), viewing the scene (up_where) as well as site-specific screening (real_where), which represents the record number of id, showing scenes show the type of movie up_where indicating up_type plays a key role in the design[2].

Page Design and Implementation

"Carousel": the home after ChinaVEC platform micro cinema navigation add up, click into the micro cinema home, first of all you see is the four $800 \times 286$ pixel size of JPG image stills showing a structure composed carousel. These four stills were called from the database, they both automatic 
switching, but also how to manually click the bottom right corner of the four-point switch. Add and modify its image as a realization of the code substantially the same as the code :( four figures)

\section{$<$ a target="_blank" $><$ img src="imgs/p1.jpg" width="970px" \\ title="the great wall" alt="the great wall" $\mid></ \mathrm{a}>$}

"Tab label": at the bottom of the carousel figure four different contents and from top to bottom in order of priority the tab label and four div their respective subordinates. Its function is to show the film classification, there are two classification basis, the first large-classified according to the film's screening scene, each category then sub-classified based on the film type. The film is divided into sub-classification record, youth, urban and rural type IV, while showing a scene divided into four categories: entertainment: cafes, bars or the like teahouse leisure activities and board games bar, chess room, KTV and other entertainment place; Square lithography: public screenings micro-film, similar to a large plaza and mall frequency screen, subway and bus excursions and other mobile media; campus micro Cinema: school choice classroom, auditorium or gymnasium to be micro-film screenings, screenings The specific time and specific location is not fixed; the journey on demand: The resort, farmhouse, on rent, travel or prolonged sitting in this category of aircraft and high-speed rail locations group gatherings and travel tours. [3] The next tab tag title information for each category is arranged to call out the extend of data from the database table, which call code:

\section{\$sql = "SELECT * from `extend where ‘up_type`='recode' AND \\ 'up_where`= 'entertainment' limit 4";}

Code displays similar calls were carried out according to the type of movie "up_type" and show scenes "up_where" two words, and use "limit 4" to limit the number of records calls to the database. Each call record does not need to write code once each individually, just use a "while loop" button, as follows:

\section{\$result=mysql_query(\$sql); \\ while(\$row=mysql_fetch_array(\$result))}

"With the same type of film sets the scene": In the lower right corner of each tab label can see there is a div called "more" jump link, click on the "more", it will jump to a new page to display the same type in the same scene all videos. Because under the tab label home movie only shows four, it is necessary to re-design a page that displays all of the movie. [4]Since large number of films, so do the pagination settings here, in the lower left corner after displaying video images and a return jump button, click on the return that is immediately jump to the micro-cinema home. Compared calling movie information tab label only limit the number of records less code:

\section{\$sql="SELECT * from `extend where ‘up_type ='youth'}

AND where `up_where`='travel' ";

"Single-film breakdown": Under the tab label or with the same type of movie scenes movie pictures or videos focus click the name under the picture will jump to a special presentation of the film details the content of the page, the page is referred to herein as "single unit movie details "page. [5]This page contains the film's Chinese name, English name, director, producer, film briefly, film type, showing the scene, showing the specific time, showing a total of nine specific location content, and display video image. To achieve this jump page must first get the film in the database id number,After obtaining the id number, then we must pass the data from the database id number is called,Then it is to call the id number corresponding to the specific data of the movie,And then went on to call the film's picture,Thus, the film details the entire single film detail page on the show.

\section{Micro-cinema system module design and implementation Admin}

\section{Micro-cinema system module Admin section Introduction}

The design of the micro-cinema background management section also in ChinaVEC background management system established in its portal management this item, named "micro-cinema management", under the "Cinema sets" Editable item. The micro-cinema system background management system for movie information have any changes in the database (extend data sheet) will also change together. Background management system throughout the movie and its message is 
in accordance with their id number in the database in reverse order, so that the arrangement is conducive to add operation:

\section{\$sql = "SELECT * from `extend`order by id desc limit \$start_num, \\ \$page_num";}

Which "by id desc" is meant by id number in reverse order. Because there were too many movies, the data also through "while loop" call out.

\section{Background Management System Features}

"Add": First, open the micro cinema background control page, click on the top right corner of the blue "Add", the new record will be out of the edit page, follow the prompts to fill in the information below and then click "Submit", there will be play box prompts successful entry. The new record entry, in fact, is to insert new data into the database:

$$
\begin{aligned}
& \text { \$sql = "INSERT INTO 'chinavec'.'extend } \\
& \text { ('director ', ’producer`, 'title_cn`, 'title_en`, 'dscrp’, } \\
& \text { 'up_type', 'up_time`, 'up_where', 'real_where`) } \\
& \text { VALUES ('\$director' ,'\$producer' ,'\$title_cn' ,'\$title_en' , } \\
& \text { '\$dscrp', '\$up_type', '\$up_time', '\$up_where', '\$real_where');"; }
\end{aligned}
$$

"Delete": delete the message There are two ways, one is the direct point deletion; the second is to choose Delete. Just click directly delete each record that is the right of the "Delete", the system will pop up to confirm the deletion which records prompt window, click OK and then automatically alert message is deleted successfully, click OK again, the system will automatically refresh records, delete records has disappeared, deleted successfully. Select Delete this record was at the far right of each record check, you can also check a few records, you can also click on the new left of the "Select All / Clear All", then select the lower left of the page click Delete option button, the same continuous click determined automatically prompted to delete. After the record is deleted unrecoverable delete key code:

$$
\text { \$sql="DELETE FROM 'extend WHERE id`='\$validid1"'; }
$$

"Edit": This one is recording editing information for each record to the right of the Edit button to enter the edit page, you can make any modifications to the page, click Submit change after completing the system will automatically pop success message editor window, after clicking OK the system will automatically jump back and refresh the information after the update, the revised update of the data in a database.

"Search": search box search keywords content and movie screenings scene types. Fill in the box keyword, such as "country", click Search, the system will automatically display a rustic type of record information, 15 records per page.

Related key code, obtain the keyword keyword:

$$
\text { \$keyword = \$_GET['q']; }
$$

And then transferred from the database associated with the scene or type of data:

\$sql = "SELECT * from `extend where up_type` like '\%

".\$keyword."\%' or `up_where` like '\%".\$keyword."\%

'LIMIT \$start_num, \$page_num";

\section{References}

[1] Google, www.google.com

[2] M. Flickner, H. Sawhney, W. Niblack, J. Ashley, Q. Huang,B. Dom, M. Gorkani, J. Hafner, D. Lee, D. Petkovic, D.Steele and P. Yanker, "Query by Image and Video Content:

the QBIC System”, IEEE Computer Magazine, pp. 23-32,Sept. 1995.

[3] T. Gevers and A. W. M. Smeulders, "PicToSeek: CombiningColor and shape Invariant Features for Image Retrieval,"IEEE Trans. on Image Processing, Vol. 9, No. 1, pp. 102- 
119, January 2000.

[4] A. Hamrapur, A. Gupta, B. Horowitz, C. F. Shu, C. Fuller, J.Bach, M. Gorkani and R. Jain, "Virage Video Engine," SPIEProc. Storage and Retrieval for Video and Image DatabasesV, pp. 188-197, San Jose, CA, Feb. 1997.

[5] A. Doulamis, N. Doulamis, and S. Kollias, "A fuzzy videocontent representation for video summarization and contentbasedretrieval," Signal Processing, Vol. 80, pp. 1049-1067,

June 2000. 